SISSA-ISAS 57/93/EP

ASITP 93-25

hep-th/9305005

\title{
Multi-field representations of the KP hierarchy and multi-matrix models
}

\author{
L.Bonora \\ International School for Advanced Studies (SISSA/ISAS) \\ Via Beirut 2, 34014 Trieste, Italy \\ INFN, Sezione di Trieste. \\ C.S.Xiong \\ Institute of Theoretical Physics, Academia Sinica \\ P.O.Box 2735, Beijing 100080, China
}

\begin{abstract}
We discuss the integrable hierarchies that appear in multi-matrix models. They can be envisaged as multi-field representations of the KP hierarchy. We then study the possible reductions of this systems via the Dirac reduction method by suppressing successively one by one part of the fields. We find in this way new integrable hierarchies, of which we are able to write the Lax pair representations by means of suitable Drinfeld-Sokolov linear systems. At the bottom of each reduction procedure we find an $N$-th $\mathrm{KdV}$ hierarchy. We discuss in detail the case which leads to the KdV hierarchy and to the Boussinesque hierarchy, as well as the general case in the dispersionless limit.
\end{abstract}




\section{Introduction}

In [1] we showed that in multi-matrix models there naturally appear pseudo-differential operators of the type (up to some relabelling)

$$
L=\partial+\sum_{l=1}^{N-1} a_{l} \frac{1}{\partial-S_{l}} \frac{1}{\partial-S_{l-1}} \cdots \frac{1}{\partial-S_{1}}
$$

where $\partial=\frac{\partial}{\partial x}=\frac{\partial}{\partial t_{1,1}}$ and $a_{1}, \ldots, a_{N-1}, S_{1}, \ldots, S_{N-1}$ are independent coordinates (fields) of the system (throughout the paper, when we write $N$, we refer precisely to this equation). They appear in linear systems of the type

$$
\begin{aligned}
& L \Psi=\lambda \Psi \\
& \frac{\partial}{\partial t_{r}} \Psi=\left(L^{r}\right)_{+} \Psi, \quad r=1,2,3, \ldots
\end{aligned}
$$

whose consistency conditions are

$$
\frac{\partial}{\partial t_{r}} L=\left[\left(L^{r}\right)_{+}, L\right]
$$

Here $\lambda$ is the spectral parameter and the $t_{r} \equiv t_{1, r}$ 's are, for example, the self-coupling parameters of the first matrix in the model. In the two above equations, the label + appended to a pseudodifferential operator represents the purely differential part of it.

In [1] we proved that multi-matrix models are exactly represented by the solutions of the above integrable hierarchies of equations that moreover satisfy suitable string equations (we do not write them down here since they do not enter the subsequent discussion). This sets the problem of classifying the various distinct integrable hierarchies that come up in this way and, subsequently, the problem of identifying these hierarchies with topological models of $2 \mathrm{~d}$ gravity plus matter, just as we did in [2] for one-matrix models. Here we will be dealing only with the first problem: precisely we want not only to describe the integrable hierarchies of the type (4), but also those that can be obtained from them via reduction.

The main result of this paper can be summarized as follows: 1) for each $N$ in (1) we find $N$ distinct integrable hierarchies which are obtained by suppressing successively the fields $S_{l}$; 2) of each such hierarchy we write down a Drinfeld-Sokolov linear system from which the Lax pair representation can be easily extracted; 3) it is irrelevant what fields we suppress first, the hierarchies with the same number of $S$ fields being isomorphic; 4) at the end of this cascade procedure we find the $N$-th $\mathrm{KdV}$ hierarchy. This is likely to represent a complete classification of all the integrable hierarchies (with a finite number of fields) appearing in multi-matrix models.

As for the reduction procedure, it is not simply a restriction on the flow equations (as we did in [2] for the NLS hierarchy), but a true reduction of the Hamiltonian system where the vanishing of the $S$ fields is imposed as a second class constraint and leads to the introduction of the corresponding Dirac brackets $\emptyset$.

We remark that this result is, in particular, a proof of the conjecture that multi-matrix models do lead to $N$-th KdV hierarchies, [3]. One should also add that the result is interesting independently of multi-matrix models: in fact the hierarchy (2, (1) is an integrable restriction of

\footnotetext{
${ }^{1}$ Thoughout the paper we keep this technical distinction: restriction means imposing some constraints on the equations of motion, while reduction means Hamiltonian reduction.
} 
the KP hierarchy which goes under the name of multi-field representation of the KP hierarchy. The properties of the multi-field representations of the KP hierarchy, not to speak of their reductions, have not received thus far a large attention, see however [4], [6], [5], [0] . But, needless to say, for us the main motivation to study these problems came from multi-matrix models.

The letter is organized as follows. In section 2 we rediscuss the reduction from the NLS hierarchy to the KdV hierarchy as a prototype of the subsequent reductions. In section 3 we discuss at length the system corresponding to $N=3$ and in section 4 its integrable reduction. In section 5 we present a compact way to describe all the integrable systems of the type (1) together with their integrable reductions. In section 6 we discuss the dispersionless limit of the system (1) with generic $N$ and its integrable reduction, which turn out to be the dispersionless limit of the $N$-th KdV hierarchy.

To end this section let us introduce a few definitions. Throughout the paper we come across integrable systems which can be given a Lax pair representation, i.e. they can be formulated by means of a differential or pseudo-differential operator of the form

$$
A=\partial^{k}+a \partial^{k-2}+b \partial^{k-3}+\ldots
$$

The corresponding flows then are given by

$$
\frac{\partial}{\partial t_{r}} A=\left[A_{+}^{\frac{r}{k}}, A\right]
$$

We define

$$
<A>=\int d x a_{-1}(x), \quad A=\ldots+a_{-1}(x) \partial^{-1}+\ldots
$$

and for any differential operator $X$ we set $f_{X}(A)=<A X>$. Two Poisson brackets are then given by

$$
\begin{aligned}
\left\{f_{X}, f_{Y}\right\}_{1}(A)= & <A[Y, X]_{R}> \\
\left\{f_{X}, f_{Y}\right\}_{2}(A)= & <(X A)_{+} Y A>-<(A X)_{+} A Y>+ \\
& +\frac{1}{k} \int[A, Y]_{(-1)}\left(\partial^{-1}[A, X]_{(-1)}\right)
\end{aligned}
$$

In the first bracket, [8], [9],

$$
[X, Y]_{R}=\frac{1}{2}([R X, Y]+[X, R Y]), \quad R X=X_{+}-X_{-}, \quad X_{-}=X-X_{+}
$$

and in the second bracket the label $(-1)$ means the coefficient of $\partial^{-1}$. We can further introduce the Hamiltonians via

$$
H_{r}=\frac{k}{r}<A^{\frac{r}{k}}>, \quad \forall k \geq 1
$$

Integrability of the system is guaranteed since these quantities are in involution and the Poisson brackets are compatible, i.e.

$$
\left\{H_{r+k}, f\right\}_{1}=\left\{H_{r}, f\right\}_{2} \quad \text { for any function } f
$$

In particular the flow equations are given by

$$
\frac{\partial}{\partial t_{r}} f=\left\{f, H_{r}\right\}_{2}=\left\{f, H_{r+k}\right\}_{1}
$$




\section{Reduction of the NLS hierarchy}

To illustrate our method we rederive here the reduction of the NLS hierarchy to the KdV hierarchy. One Hermitean matrix models with the most general potential are exactly described by the NLS hierarchy [10, [2].

The NLS hierarchy can be defined via the Lax operator 9

$$
L=\partial+R \frac{1}{\partial-S}
$$

Now, following the prescription of the previous section, we can calculate anything we need. In particular the first Poisson brackets are

$$
\begin{aligned}
& \{R(x), R(y)\}_{1}=0 \quad\{S(x), S(y)\}_{1}=0 \\
& \{R(x), S(y)\}_{1}=-\partial_{x} \delta(x-y)
\end{aligned}
$$

and the second ones are

$$
\begin{aligned}
& \{R(x), R(y)\}_{2}=\left(2 R \partial+R^{\prime}\right) \delta(x-y) \\
& \{S(x), S(y)\}_{2}=2 \partial \delta(x-y) \\
& \{R(x), S(y)\}_{2}=\left(S \partial+\partial^{2}\right) \delta(x-y)
\end{aligned}
$$

Henceforth we will use for Poisson brackets the simplified notation of (12) where the fields and derivatives (denoted also by a prime) appearing in RHS are understood to be evaluated at $x$.

The Hamiltonians are

$$
H_{1}=\int d x R, \quad H_{2}=\int d x R S, \quad H_{3}=\int d x\left(R^{2}+R S^{2}+R^{\prime} S\right), \quad \text { etc. }
$$

and the flow equations are easy to compute. For example the $t_{2}$ flow equations are:

$$
\frac{\partial S}{\partial t_{2}}=-S^{\prime \prime}+2 S^{\prime} S+2 R^{\prime}, \quad \frac{\partial R}{\partial t_{2}}=-3 R^{\prime \prime}+2(R S)^{\prime}
$$

which is a disguised form of the non-linear Schrödinger equation.

Let us study now the reduction

$$
S=0
$$

In [2] we considered a restriction $S=0$ applied only to the flow equations and we ended up with the $\mathrm{KdV}$ hierarchy. However the restriction method, tough valid in this case, leads to inconsistent results when $N>2$ in (11), [1]. We have to consider the reduction of the Hamiltonian system. The constraint $S=0$ is second class and we have to follow Dirac's procedure, 11]. For the second Poisson brackets we can introduce the corresponding Dirac bracket in the reduced system

$$
\{R(x), R(y)\}_{2 D}=\left(2 R \partial+R^{\prime}+\frac{1}{2} \partial^{3}\right) \delta(x-y)
$$

If we use this and the constrained $H_{3}$ Hamiltonian we obtain

$$
\frac{\partial}{\partial t_{3}} R=R^{\prime \prime \prime}+6 R R^{\prime}
$$

\footnotetext{
${ }^{2}$ We remark that this differs from the Lax operator used in [2] by the redefinition $S \rightarrow S+(\ln R)^{\prime}$. We adopt the definition (9) in order to conform to the general definition (11) - this is the case $N=2$ with $a_{1}=R$ and $S_{1}=S$.
} 
which is the $\mathrm{KdV}$ equation.

The first Poisson bracket is not reducible. So we have to look for another Poisson bracket for the reduced system. It is not difficult to find it as well as the series of Hamiltonians using the fact that the two Poisson brackets must be compatible and the Hamiltonians in involution. This is however a long procedure. There is a much quicker method, which consists in finding a Lax pair representation. For this particular case the solution is universally known: the Lax operator is (up to a rescaling of the flow parameters)

$$
\partial^{2}+R
$$

With this and the formulas of the introduction we can calculate Poisson brackets, Hamiltonians and flows (i.e. the KdV hierarchy) of the reduced system.

This suggests us a general method to study the reductions of the more complex systems with $N>2$. We will impose restriction constraints which will turn out to be second class. We will compute the Dirac brackets corresponding to the second Poisson structure and find the equations of motion of the reduced system. It will be then easy to identify the appropriate Lax operator.

\section{$3 \quad$ Four-field representation of the KP hierarchy}

The simplest integrable system that appear in matrix models after the NLS system is the fourfield restriction of the KP hierarchy. It naturally leads, via reduction, to the Boussinesque hierarchy. Let us describe this system in some detail.

We begin with the following set of differential equations

$$
\begin{aligned}
& \frac{\partial}{\partial t} a_{1}=a_{1}^{\prime \prime}+2 a_{2}^{\prime}+2\left(a_{1} S_{1}\right)^{\prime} \\
& \frac{\partial}{\partial t} a_{2}=a_{2}^{\prime \prime}+2 a_{2}^{\prime} S_{2}+2 a_{2}\left(S_{1}+S_{2}\right)^{\prime} \\
& \frac{\partial}{\partial t} S_{1}=2 a_{1}^{\prime}+2 S_{1} S_{1}^{\prime}-S_{1}^{\prime \prime} \\
& \frac{\partial}{\partial t} S_{2}=2 a_{1}^{\prime}+2 S_{2} S_{2}^{\prime}-S_{2}^{\prime \prime}-2 S_{1}^{\prime \prime}
\end{aligned}
$$

We want to show that these equations do define an integrable system, i.e. they possess a biHamiltonian structure.

\subsection{The Poisson Brackets}

It is not difficult to show that these equations can be written as Hamiltonian equations

$$
\frac{\partial}{\partial t} f=\{f, H\}_{1}
$$

where the function $f$ stands for $\left(a_{1}, a_{2}, S_{1}, S_{2}\right)$, and the Hamiltonian takes the following form

$$
H=\int d x\left[a_{1} S_{1}^{2}-a_{1} S_{1}^{\prime}+a_{2}\left(S_{1}+S_{2}\right)+a_{1}^{2}\right]
$$


The Poisson brackets are

$$
\begin{aligned}
& \left\{a_{1}(x), S_{1}(y)\right\}_{1}=\delta^{\prime}(x-y), \quad\left\{a_{1}(x), S_{2}(y)\right\}_{1}=\delta^{\prime}(x-y), \\
& \left\{a_{2}(x), a_{2}(y)\right\}_{1}=\left(2 a_{2} \partial+a_{2}^{\prime}\right) \delta(x-y), \\
& \left\{a_{2}(x), S_{2}(y)\right\}_{1}=\left(\partial^{2}+\left(S_{2}-S_{1}\right) \partial\right) \delta(x-y) .
\end{aligned}
$$

All the others vanish. One can check that these Poisson brackets satisfy the Jacobi identity.

The important thing is that eq.(16) admits another Hamiltonian representation, that is

$$
\frac{\partial}{\partial t} f=\{f, \tilde{H}\}_{2}
$$

with

$$
\tilde{H}=\int d x\left[a_{1} S_{1}+a_{2}\right]
$$

And the Poisson brackets take the form

$$
\begin{aligned}
& \left\{a_{1}(x), a_{1}(y)\right\}_{2}=\left(2 a_{1} \partial+a_{1}^{\prime}\right) \delta(x-y), \quad\left\{a_{1}(x), a_{2}(y)\right\}_{2}=\left(3 a_{2} \partial+2 a_{2}^{\prime}\right) \delta(x-y), \\
& \left\{a_{1}(x), S_{1}(y)\right\}_{2}=\left(\partial^{2}+S_{1} \partial\right) \delta(x-y), \quad\left\{a_{1}(x), S_{2}(y)\right\}_{2}=\left(2 \partial^{2}+S_{2} \partial\right) \delta(x-y), \\
& \left\{a_{2}(x), a_{2}(y)\right\}_{2}=\left[\left(2 a_{2}^{\prime}+4 a_{2} S_{2}-2 a_{2} S_{1}\right) \partial+a_{2}^{\prime \prime}+\left(2 a_{2} S_{2}-a_{2} S_{1}\right)^{\prime}\right] \delta(x-y), \\
& \left\{a_{2}(x), S_{2}(y)\right\}_{2}=\left(a_{1} \partial+\left(\partial+S_{2}\right)\left(\partial+S_{2}-S_{1}\right) \partial\right) \delta(x-y), \\
& \left\{S_{1}(x), S_{1}(y)\right\}_{2}=2 \delta^{\prime}(x-y), \quad\left\{a_{2}(x), S_{1}(y)\right\}_{2}=0, \\
& \left\{S_{1}(x), S_{2}(y)\right\}_{2}=\delta^{\prime}(x-y), \quad\left\{S_{2}(x), S_{2}(y)\right\}_{2}=2 \delta^{\prime}(x-y),
\end{aligned}
$$

It is straightforward to check that the Jacobi identities are satisfied. Moreover

$$
\{H, \tilde{H}\}=0
$$

for both brackets. Therefore we have shown that the system (16) has a bi-Hamiltonian structure.

\subsection{Lax pair representation}

In principle we can use the two compatible Poisson brackets to construct all the conserved quantities, the first few are

$$
H_{1}=\int d x a_{1}, \quad H_{2}=\tilde{H}, \quad, H_{3}=H, \ldots
$$

In turn these conserved quantities generate new flows. The first ones are trivial, the second flows are eq.(16), and the third ones take the following form

$$
\begin{aligned}
\frac{\partial}{\partial t_{3}} a_{1} & =\left[3 a_{2}^{\prime}+a_{1}^{\prime \prime}+3 a_{1}^{2}+3 a_{1}^{\prime} S_{1}+3 a_{1} S_{1}^{2}+3 a_{2}\left(S_{1}+S_{2}\right)\right]^{\prime} \\
\frac{\partial}{\partial t_{3}} a_{2} & =\left[a_{2}^{\prime \prime}+3 a_{2}^{\prime} S_{2}+3 a_{2} S_{2}^{2}\right]^{\prime}+6 a_{1}^{\prime} a_{2}+3 a_{1} a_{2}^{\prime}+3 a_{2}\left(S_{1}+S_{2}\right) S_{1}^{\prime}-3 a_{2} S_{1}^{\prime \prime} \\
\frac{\partial}{\partial t_{3}} S_{1} & =\left[3 a_{2}+6 a_{1} S_{1}+S_{1}^{\prime \prime}+S_{1}^{3}-3 S_{1} S_{1}^{\prime}\right]^{\prime} \\
\frac{\partial}{\partial t_{3}} S_{2} & =\left[3 a_{2}-3 a_{1}^{\prime}+3 a_{1}\left(S_{1}+S_{2}\right)+S_{2}^{\prime \prime}+3 S_{1}^{\prime \prime}+S_{2}^{3}-3 S_{2} S_{2}^{\prime}-3\left(S_{1}+S_{2}\right) S_{1}^{\prime}\right]^{\prime}
\end{aligned}
$$


However there is a more way to proceed. It is by means of the Lax pair representation. Consider

$$
L=\partial+a_{1} \frac{1}{\partial-S_{1}}+a_{2} \frac{1}{\partial-S_{2}} \frac{1}{\partial-S_{1}} .
$$

Then not only can the above equations of motion be recast into

$$
\frac{\partial}{\partial t_{r}} L=\left[L_{+}^{r}, L\right], \quad r \geq 1 .
$$

with the second flow $(r=2)$ giving exactly eq.16) with $t=t_{2}$ and the third flow $(r=3)$ giving the above equation with $t=t_{3}$, but, more important, we can compute all the Poisson brackets, Hamiltonians and flows by means of the formulas given in the introduction. The operator (24) is nothing but our initial operator (1) taken from multi-matrix models when $N=3$.

Comparing with the ordinary coordinates of KP hierarchy

$$
L=\partial+\sum_{l=1}^{\infty} u_{l} \partial^{-l}
$$

we see that our integrable system is nothing but a particular restriction of the KP hierarchy, the restriction conditions being

$$
\begin{aligned}
& u_{1}=a_{1}, \quad u_{2}=a_{2}+a_{1} S_{1} \\
& u_{l+2}=a_{1}\left(-\partial+S_{1}\right)^{l+1} \cdot 1+a_{2} \sum_{k=0}^{l}\left(-\partial+S_{2}\right)^{k}\left(-\partial+S_{1}\right)^{l-k} \cdot 1 . \quad l \geq 0
\end{aligned}
$$

So we may say that our system (25) is nothing but the four field representation of the KP hierarchy.

\section{$3.3 \quad W_{\infty}$-algebra}

Since the new hierarchy is a restricted KP hierarchy we would expect that the KP coordinates $u_{l}$ 's defined in eq.(27) to form a $W_{\infty}$ algebra with respect to two Poisson brackets (19) and (22) (see in this regard, 12, 13, 14]). This is actually the case. We give here only the result with respect to the first Poisson bracket.

With respect to the coordinates $u_{l}$ of eq.(27)

$$
\left\{u_{i+2}(x), u_{j+2}(y)\right\}_{1}=\left[\sum_{l=0}^{j+1}\left(\begin{array}{c}
j+1 \\
l
\end{array}\right) \partial^{l} u_{i+j-l+3}-\sum_{l=0}^{i+1}(-1)^{l}\left(\begin{array}{c}
i+1 \\
l
\end{array}\right) u_{i+j-l+3} \partial^{l}\right] \delta(x-y)
$$

Therefore the $u_{i}$ 's form a $W_{1+\infty}$-algebra, which we call the four-field representation of such an algebra.

\section{Reductions of the four-field KP hierarchy}

\subsection{First reduction}

Let us consider the possible reductions of the integrable system introduced in the previous section. We will first impose the reduction

$$
S_{1}=0
$$


With respect to the second Poisson structure (22) this is a second class constraint. However $\left\{S_{1}(x), S_{1}(y)\right\}$ has an inverse. We can therefore proceed according to Dirac and define improved Poisson brackets. These are

$$
\begin{aligned}
& \left\{a_{1}(x), a_{1}(y)\right\}_{2 D}=\left(2 a_{1} \partial+a_{1}^{\prime}+\frac{1}{2} \partial^{3}\right) \delta(x-y) \\
& \left\{a_{1}(x), a_{2}(y)\right\}_{2 D}=\left(3 a_{2} \partial+2 a_{2}^{\prime}\right) \delta(x-y) \\
& \left\{a_{1}(x), S_{2}(y)\right\}_{2 D}=\left(\frac{3}{2} \partial^{2}+S_{2} \partial\right) \delta(x-y) \\
& \left\{a_{2}(x), a_{2}(y)\right\}_{2 D}=\left[\left(2 a_{2}^{\prime}+4 a_{2} S_{2}\right) \partial+a_{2}^{\prime \prime}+2\left(a_{2} S_{2}\right)^{\prime}\right] \delta(x-y) \\
& \left\{a_{2}(x), S_{2}(y)\right\}_{2 D}=\left(a_{1} \partial+\left(\partial+S_{2}\right)^{2} \partial\right) \delta(x-y) \\
& \left\{S_{2}(x), S_{2}(y)\right\}_{2 D}=\frac{3}{2} \delta^{\prime}(x-y)
\end{aligned}
$$

Now the reduced Hamiltonian is a restriction of the original Hamiltonian (21) to the constrained manifold. Consequently the equations of motion of the constrained system are

$$
\begin{aligned}
& \frac{\partial}{\partial t} a_{1}=2 a_{2}^{\prime} \\
& \frac{\partial}{\partial t} a_{2}=a_{2}^{\prime \prime}+2\left(a_{2} S_{2}\right)^{\prime} \\
& \frac{\partial}{\partial t} S_{2}=a_{1}^{\prime}+2 S_{2} S_{2}^{\prime}-S_{2}^{\prime \prime}
\end{aligned}
$$

The first Poisson bracket (19) is not reducible. One can then proceed to search for another Poisson structure, as we did at the beginning of the previous section. One finds that with the following choice

$$
\left\{a_{1}(x), a_{1}(y)\right\}_{1^{\prime}}=2 \partial \delta(x-y), \quad\left\{a_{2}(x), S_{2}(y)\right\}_{1^{\prime}}=\partial \delta(x-y) .
$$

and with the Hamiltonian

$$
H^{\prime}=\int d x\left(a_{2}\left(S_{2}^{2}-S_{2}^{\prime}\right)+a_{1} a_{2}\right)
$$

one obtains the same equations of motion (30) and the two Hamiltonians commute. However, as we have repeatedly noticed, there is a much quicker and powerful way to see the integrability of the reduced system. In fact we can write down a Lax pair for it. The latter is based on the following pseudo-differential operator

$$
\tilde{L}=\partial^{2}+a_{1}+a_{2} \frac{1}{\partial-S_{2}}
$$

By means of the formulas in the introduction we can compute the corresponding Poisson brackets, Hamiltonians and flow equations. Among the latter we find all the above results. We conclude that the reduced system is integrable 5 .

Next we consider another possible reduction. Instead of $S_{1}=0$ we impose

$$
S_{2}=0
$$

\footnotetext{
${ }^{3}$ Via the transformation $\partial \rightarrow \partial+S_{2}$ we obtain from (33) an operator which was considered in [4]
} 
We proceed in the same way as above and we quickly realize that the reduced system we obtain is nothing but a redefinition of the first reduced system just defined. In fact if, starting from it, we make the field redefinitions

$$
S_{2} \rightarrow S_{1}, \quad a_{1} \rightarrow a_{1}+S_{1}^{\prime}, \quad a_{2} \rightarrow a_{2}+a_{1} S_{1}+S_{1}^{\prime \prime}+S_{1} S_{1}^{\prime}
$$

we find the $S_{2}=0$ reduced system, which, consequently, is the same as the first one.

Next one could wonder whether another reduction is possible, say

$$
S_{1}=S_{2}
$$

This reduction can be carried out, the Dirac bracket defined and the equations of motion derived. However there does not seem to exist any bi-Hamiltonian structure for this system. We will henceforth ignore it.

\subsection{Further reduction. The Boussinesque hierarchy.}

In the previous subsection we have found one distinct integrable reduction of the four-field KP hierarchy to a three field hierarchy. Here we further reduce the reduced hierarchy by suppressing the remaining $S$ field

$$
S_{2}=0
$$

With Dirac's procedure we find from (29)

$$
\begin{aligned}
& \left\{a_{1}(x), a_{1}(y)\right\}_{2 D^{\prime}}=\left(2 a_{1} \partial+a_{1}^{\prime}+2 \partial^{3}\right) \delta(x-y) \\
& \left\{a_{1}(x), a_{2}(y)\right\}_{2 D^{\prime}}=\left(3 a_{2} \partial+2 a_{2}^{\prime}-\partial^{2} a_{1}+\partial^{4}\right) \delta(x-y) \\
& \left\{a_{2}(x), a_{2}(y)\right\}_{2 D^{\prime}}=\left[2 a_{2}^{\prime} \partial+a_{2}^{\prime \prime}-\frac{2}{3}\left(a_{1}+\partial^{2}\right)\left(\partial a_{1}+\partial^{3}\right)\right] \delta(x-y),
\end{aligned}
$$

This is nothing but the $W_{3}$ algebra. The corresponding equations of motion calculated from the doubly constrained Hamiltonian (21) are

$$
\frac{\partial}{\partial t} a_{1}=2 a_{2}^{\prime}-a_{1}^{\prime \prime}, \quad \frac{\partial}{\partial t} a_{2}=a_{2}^{\prime \prime}-\frac{2}{3}\left(a_{1} a_{1}^{\prime}+a_{1}^{\prime \prime}\right)
$$

This is known as the Boussinesque equation and it is the first of an integrable hierarchy of equations which can be given a Lax pair representation by means of the operator

$$
\tilde{\tilde{L}}=\partial^{3}+a_{1} \partial+a_{2}
$$

We remark that starting from the four-field representation of the KP hierarchyand performing the simultaneous reduction $S_{1}=0=S_{2}$ we end up with the same system (37).

In conclusion as a result of the successive reduction of the four-field KP hierarchy we find two more distinct integrable hierarchy. The last one is the well-known Boussinesque hierarchy. 


\section{A DS representation of the previous systems.}

The integrable models and reductions we have been considering so far can be synthesized in a very compact and useful form that lends itself to generalization via suitable Drinfeld-Sokolov (DS) linear systems. From them one can easily extract the corresponding Lax pair. Let us start with the linear system

$$
\left(\begin{array}{cc}
\partial-S & -1 \\
R & \partial-\lambda
\end{array}\right)\left(\begin{array}{c}
\psi_{1} \\
\psi
\end{array}\right)=0
$$

From this it is elementary to see

$$
\left(\partial+R \frac{1}{\partial-S}\right) \psi=\lambda \psi
$$

from which we recognize the spectral equation for the NLS Lax operator studied in section 2.

Similarly

$$
\left(\begin{array}{cc}
\partial & -1 \\
R-\lambda & \partial
\end{array}\right)\left(\begin{array}{c}
\psi \\
\psi_{1}
\end{array}\right)=0, \quad \text { and } \quad\left(\partial^{2}+R\right) \psi=\lambda \psi
$$

The last is nothing but the spectral equation for the KdV Lax operator.

Let us pass now to the systems with $N=3$ studied in section 3 . We have

$$
\left(\begin{array}{ccc}
\partial-S_{2} & -1 & 0 \\
0 & \partial-S_{1} & -1 \\
a_{2} & a_{1} & \partial-\lambda
\end{array}\right)\left(\begin{array}{c}
\psi_{1} \\
\psi_{2} \\
\psi
\end{array}\right)=0
$$

so that

$$
\left(\partial+a_{1} \frac{1}{\partial-S_{1}}+a_{2} \frac{1}{\partial-S_{2}} \frac{1}{\partial-S_{1}}\right) \psi=\lambda \psi
$$

Next

$$
\left(\begin{array}{ccc}
\partial-S_{2} & -1 & 0 \\
0 & \partial & -1 \\
a_{2} & a_{1}-\lambda & \partial
\end{array}\right)\left(\begin{array}{c}
\psi_{1} \\
\psi \\
\psi_{2}
\end{array}\right)=0, \quad \text { and } \quad\left(\partial^{2}+a_{1}+a_{2} \frac{1}{\partial-S_{2}}\right) \psi=\lambda \psi
$$

Finally

$$
\left(\begin{array}{ccc}
\partial & -1 & 0 \\
0 & \partial & -1 \\
a_{2}-\lambda & a_{1} & \partial
\end{array}\right)\left(\begin{array}{c}
\psi \\
\psi_{1} \\
\psi_{2}
\end{array}\right)=0, \quad \text { and } \quad\left(\partial^{3}+a_{1} \partial+a_{2}\right) \psi=\lambda \psi
$$

We recognize the Lax operators $L, \tilde{L}$ and $\tilde{\tilde{L}}$ of section 3 . We notice that of all the above DS linear systems, only (40) and (44) are in the standard form 115].

On the basis of these examples it is easy to extend this to a generic $N$. The initial system is

$$
\left(\begin{array}{cccccc}
\partial-S_{N-1} & -1 & 0 & \cdots & 0 & 0 \\
0 & \partial-S_{N-2} & -1 & \cdots & 0 & 0 \\
\cdots & \cdots & \cdots & \cdots & \cdots & \cdots \\
0 & 0 & 0 & \cdots & \partial-S_{1} & -1 \\
a_{N-1} & a_{N-2} & a_{N-3} & \cdots & a_{1} & \partial-\lambda
\end{array}\right)\left(\begin{array}{c}
\psi_{1} \\
\psi_{2} \\
\cdots \\
\psi_{N-1} \\
\psi
\end{array}\right)=0
$$


From this we obtain the equation $L \psi=\lambda \psi$ where $L$ is the same Lax operator as in eq.(11). The other systems can be obtained by: 1$)$ moving $-\lambda$ by one place to the left in the last row, 2) suppressing the surviving S field with lowest index, 3) making an elementary cyclic rotation upward of the vector entries, and 4) extracting the equation for $\psi$.

We obtain in such a way $N-1$ more Lax operators. The obvious conjecture is that these represent the $N-1$ distinct integrable reductions of the system defined by (1). We uniquely identify each such system with a symbol $\mathcal{S}_{N}^{k}$, where $N$ has the usual meaning, eq.(1), and $k$ counts the number of nonvanishing $S$ fields, $0 \leq k \leq N-1$. In particular the case $k=N-1$ yields the $2(N-1)$-field representation of the KP hierarchy and of the $W_{\infty}$ algebra.

The above conjecture is very plausible, but we defer the technicalities of the proof to a future publication. Instead, in this letter, we study, as additional evidence for our conjecture, the dispersionless limit of the system $\mathcal{S}_{N}^{N-1}$ and its reduction. This limit is interesting in itself, firstly because it represent a new integrable hierarchy and secondly because, in the context of matrix models, it represents the spheric limit of the theory.

\section{The dispersionless limit of $\mathcal{S}_{N}^{N-1}$}

The second flow equations of the Lax operator $L$ defined by eq.(11),

$$
\frac{\partial}{\partial t} L=\left[L_{+}^{2}, L\right]
$$

involving the fields $a_{l}, S_{l} ; 1 \leq l \leq N-1$, are

$$
\begin{gathered}
\frac{\partial}{\partial t} a_{l}=a_{l}^{\prime \prime}+2 a_{l+1}^{\prime}+2 a_{l}^{\prime} S_{l}+2 a_{l}\left(\sum_{k=1}^{l} S_{k}\right)^{\prime}, \\
\frac{\partial}{\partial t} S_{l}=2 a_{1}^{\prime}+2 S_{l} S_{l}^{\prime}-S_{l}^{\prime \prime}-2\left(\sum_{k=1}^{l-1} S_{k}\right)^{\prime \prime} .
\end{gathered}
$$

The recipe to define the dispersionless version of this integrable differential system is very simple. We discard all the higher derivatives in the equations or, alternatively, we make the substitution

$$
\begin{aligned}
& \partial \Longrightarrow p: \text { canonical momentum, } \\
& {[\partial, x]=1 \Longrightarrow\{p, x\}=1}
\end{aligned}
$$

Applying the first procedure to eq.(47), we obtain

$$
\begin{aligned}
\frac{\partial}{\partial t} a_{l} & =2 a_{l+1}^{\prime}+2 a_{l}^{\prime} S_{l}+2 a_{l}\left(\sum_{k=1}^{l} S_{k}\right)^{\prime}, \\
\frac{\partial}{\partial t} S_{l} & =2 a_{1}^{\prime}+2 S_{l} S_{l}^{\prime} .
\end{aligned}
$$

We see that all the fields $S_{l}(1 \leq l \leq N-1)$ have the same equations of motion, so we may simply identify them, and denote them $S$. Then our dispersionless equations are

$$
\begin{aligned}
& \frac{\partial}{\partial t} a_{l}=2 a_{l+1}^{\prime}+2 a_{l}^{\prime} S+2 l a_{l} S^{\prime}, \\
& \frac{\partial}{\partial t} S=2 a_{1}^{\prime}+2 S S^{\prime} .
\end{aligned}
$$


We remark that the same conclusion can be reached in full generality if we start from the matrix models [1]. This set of equations is our starting point. Hereafter we will analyse their Hamiltonian structure and the associated algebras.

\subsection{Bi-Hamiltonian structure}

First we notice that eqs. (49) can be reexpressed as Hamiltonian equations

$$
\frac{\partial}{\partial t} f=\left\{f, H_{3}\right\}_{1}=\left\{f, H_{2}\right\}_{2} \text {. }
$$

where $f$ stands for $a_{l}$ and $S$, and

$$
H_{2}=\int\left(a_{2}+a_{1} S\right) d x, \quad H_{3}=\int\left(a_{3}+2 a_{2} S+a_{1} S^{2}+a_{1}^{2}\right) d x .
$$

The first Poisson brackets are

$$
\begin{aligned}
& \left\{a_{i}(x), a_{j}(y)\right\}_{1}=\left[(i-1) a_{i+j-2} \partial+(j-1) \partial a_{i+j-2}\right] \delta(x-y), \quad 2 \leq i, j \leq N-1 . \\
& \left\{a_{1}(x), S(y)\right\}_{1}=\delta^{\prime}(x-y) .
\end{aligned}
$$

while all the other vanish.

The second Poisson brackets are

$$
\begin{aligned}
\{S(x), S(y)\}_{2}= & \frac{N}{N-1} \partial \delta(x-y), \quad\left\{S(x), a_{1}(y)\right\}_{2}=\partial S \delta(x-y) \\
\left\{S(x), a_{j}(y)\right\}_{2}= & \frac{N-j}{N-1} \partial a_{j-1} \delta(x-y), \quad 2 \leq j \leq N-1 ; \\
\left\{a_{1}(x), a_{j}(y)\right\}_{2}= & \left(a_{j}(x) \partial+j \partial a_{j}\right) \delta(x-y), \\
\left\{a_{i}(x), a_{j}(y)\right\}_{2}= & {\left[\left(i a_{i+j-1} \partial+j \partial a_{i+j-1}\right)-(i-1) \frac{N-j}{N-1} a_{i-1} \partial a_{j-1}+\right.} \\
& +\sum_{l=1}^{i-2}\left((i-l-1) a_{i+j-l-2} \partial a_{l}+(j-l-1) a_{l} \partial a_{i+j-l-2}\right)+ \\
& \left.+(i-1) a_{i+j-2} \partial S+(j-1) S \partial a_{i+j-2}\right] \delta(x-y), \quad i, j \geq 2 .
\end{aligned}
$$

One can prove that these two Poisson brackets satisfy the Jacobi identities. So we have found a new integrable system. We remark that with respect to these two Poisson brackets, each subset of fields $\left\{S, a_{1}, a_{2}, \ldots, a_{l}\right\},(l \leq N-1)$ form a subalgebra.

Once again, as was done several times before, this new system can be given a most useful Lax pair representation. We introduce the 'operator'

$$
\ell=p+\sum_{l=1}^{N-1} \frac{a_{l}}{(p-S)^{l}} .
$$

and use the second prescription (48) above. Then eqs.(49) can be written as

$$
\frac{\partial}{\partial t} \ell=\left\{\ell_{+}^{2}, \ell\right\}
$$

and, in general, we can introduce an infinite series of flows

$$
\frac{\partial}{\partial t_{r}} \ell=\left\{\ell_{+}^{r}, \ell\right\}, \quad 1 \leq r
$$




\subsection{The Dirac reduction}

The only possible reduction is in this case

$$
S=0,
$$

The improved second Poisson brackets are

$$
\begin{aligned}
\left\{a_{i}(x), a_{j}(y)\right\}_{2 D}= & {\left[i a_{i+j-1} \partial+j \partial a_{i+j-1}-i \frac{N-j}{N} a_{i-1} \partial a_{j-1}+\right.} \\
& \left.+\sum_{l=1}^{i-2}\left((i-l-1) a_{i+j-l-2} \partial a_{l}+(j-l-1) a_{l} \partial a_{i+j-l-2}\right)\right] \delta(x-y) .
\end{aligned}
$$

The reduced equations of motion are

$$
\frac{\partial a_{i}}{\partial t}=2 a_{i+1}^{\prime}-2 \frac{N-i}{N} a_{i-1} a_{1}^{\prime}
$$

The above coincide with the Poisson brackets and equations of motion of the dispersionless $N$-th KdV hierarchy. To see this, let us consider the $N$-th KdV Lax operator in the dispersionless limit

$$
\mathcal{A}=p^{N+1}+\sum_{l=1}^{N} w_{l} p^{N-l}
$$

The dispersionless equations of motion are

$$
\frac{\partial}{\partial t_{r}} \mathcal{A}=\left\{\mathcal{A}_{+}^{\frac{r}{N+1}}, \mathcal{A}\right\}
$$

The Poisson structures become

$$
\left\{w_{i}(x), w_{j}(y)\right\}_{1}=\left[(i-N) w_{i+j-N-1} \partial+(j-N) \partial w_{i+j-N-1}-N \delta_{i+j, N} \partial\right] \delta(x-y)
$$

and

$$
\begin{aligned}
\left\{w_{i}(x), w_{j}(y)\right\}_{2}= & {\left[i w_{i+j-1} \partial+j \partial w_{i+j-1}-i \frac{N-j}{N} w_{i-1} \partial w_{j-1}\right.} \\
& \left.+\sum_{l=1}^{i-2}\left((i-l-1) w_{i+j-l-2} \partial w_{l}+(j-l-1) w_{l} \partial w_{i+j-l-2}\right)\right] \delta(x-y)
\end{aligned}
$$

If we identify

$$
a_{i}=w_{i}, \quad i \geq 1
$$

we see that the last bracket coincides with (54), which therefore constitutes a realization of the $w_{N}$ algebra. The equations of motion trivially coincide.

In this way we have got another example of a reduction that ends up with an $N$-th $\mathrm{KdV}$ hierarchy, as expected on the basis of the general statements of the previous section. We finally remark that, if we start from the dispersionless limit of $\mathcal{S}_{N}^{k}$, with $0 \leq k<N-1$, and make the reduction $S=0$ we end up always with the same system, i.e. the dispersionless $N$-th $\mathrm{KdV}$ hierarchy.

Acknowledgements. One of us (C.-S. X.) would like to thank Q.P.Liu and Z.Y.Zhu for discussions and ICTP and SISSA for hospitality. 


\section{References}

[1] L.Bonora and C.S.Xiong, Multi-matrix models without continuum limit, SISSA 211/92/EP, hep-th/9212070.

[2] L.Bonora and C.S.Xiong, Matrix models without scaling limit SISSA 161/92/EP, to be published in IJMP A.

[3] M.Douglas, Phys.Lett. B238 (1990) 176.

[4] B.A.Kupersmith, Comm.Math.Phys. 99 (1985) 51.

[5] H.Aratyn, E.Nissimov, S.Pacheva and I.Vaysburd R-Matrix formulation of KP hierarchies and their gauge equivalence, CERN-TH 6627/92.

[6] W.Oevel and W.Strampp Constrained KP hierarchy and bi-Hamiltonian structures, Loughborough Univ. (Math. Rep. A168).

[7] S.Bellucci, E.Ivanov and S.Krivonos Multi-field coset realizations of $w_{1+\infty}$, LNF-92/099 (P).

[8] M.A.Semenov-Tian-Shansky, Funct.An.Appl. 17 (1983) 259.

[9] L.Dickey, Soliton equations and Hamiltonian systems, World Scientific, 1991.

[10] L.Bonora and C.S.Xiong, Phys.Lett. B285 (1992) 191.

[11] P.A.M.Dirac, Lecture notes on quantum field theory, 1965.

[12] H.Aratyn, L.A.Ferreira, J.F.Gomez and A.H.Zimerman, On two-current realization of KP hierarchy, IFT-P/020/92

[13] F.Yu and Y.-S. Wu, Phys.Rev.Lett. 68 (1992) 2996.

[14] R.Paunov, $W_{\infty}$ structure of the $\operatorname{sl}(N)$ Conformal Affine Toda Theories, SISSA 32/93/EP.

[15] V.G.Drinfeld and V.V.Sokolov, J.Sov.Math. 3019841975. 